$\xi=-1$

\title{
Performance of EEDR Routing Protocol under Various Path Loss Models for WSNs
}

\author{
Pramod M S ${ }^{1 *}$, Shivashankar ${ }^{2}$ \\ ${ }^{1}$ Assistant Professor, Department of Electronics \& Communication Engineering, Sri Venkateshwara College of Engineering, Bengaluru. \\ (email: pramod.ms97@gmail.com) \\ ${ }^{2}$ Professor and Head, Department of Electronics \& Communication Engineering, Sri Venkateshwara College of Engineering, Bengaluru. \\ *Corresponding author E-mail:chenduss123@gmail.com)
}

\begin{abstract}
Path loss is the power variation between the receiver power and the transmitter power. The attenuation of the signal takes place in Wireless Sensor Networks (WSNs) because of path loss. There are various kinds of path loss due to absorption, reflection, refraction and many other factors under diverse environments which are all defined under path loss models. This research paper provides a brief study on different path loss models and their mathematical evaluation. The objective of the proposed work is to evaluate the Energy Efficient Distributed Receiver (EEDR) based routing protocol under the various path loss models and check the performance variation in different environments. The simulation results shows the effect of path loss on end-to-end delay, number of hops, energy consumption and the number of alive nodes in WSNs topology.
\end{abstract}

Keywords: Channel Quality, Energy, Path loss, Protocol, Receiver, Sender.

\section{Introduction}

Wireless Sensor Networks (WSNs) is made of small or infrastructure-less network. The nodes monitor the environment where they are deployed and send the sensed data by working together. The number of sensor nodes deployed ranges from few tens to thousands depending on the environment. The deployment of nodes in unstructured WSN is in ad hoc way, whereas in structured WSN there is pre-planned deployment of few nodes which reduces the maintenance and cost of the network [1]. The WSN design is very challenging as the nodes have limited battery power and are expected to work for long period of time. It may be cost prohibitive or impossible to replace the batteries of the deployed sensor nodes [2]. The transmission of data with improved efficiency and reliability is the primary concern in resource constrained WSN. Most of the existing works involve retransmission of lost data packet that includes extra overhead in the network [3].

The basic property of transmission of the signal over a wireless media is that power decays with distance. It is much more composite under the terrestrial environments because of physical features such as reflections from ground, scattering, shadowing and many other factors. The WSN is densely deployed by sensor nodes and the network is irregular which causes more discrepancy in the link distances [4]. In wireless channels, the network design needs a precise estimation as there is a strong impact of path loss over the link quality. The path loss also depends on the heights of the transmitter and receiver antennas. The signal gets weaker as it travels through the propagation medium and path loss models forecast how the signals get weaker in different ways, when they travel from source to destination. Path loss models involve theoretical calculation of path loss and any study on the path loss can choose the propagation model depending on the area chosen for communication purpose. The propagation model is said to be reliable if the power requirement and coverage area is optimized by the elimination of adverse effects [5].

The wireless networks have got wide range of applications in various fields, one of the major challenges of wireless networks is obtaining high data rate and hence the requirement of larger bandwidth. The propagation channel decides the performance limit for any wireless system [6]. The implementation of the new technology in any environment requires understanding and realization of the propagation characteristics. The path loss models also decide the requirements such as design of antennas, power required for transmission and spacing between the nodes and hence any architecture development needs consideration of path loss models for performance and cost analysis [7].

In this paper, various path loss models are studied and the EEDR routing protocol [8] is evaluated for different path loss models. The performance of the protocol is evaluated considering end-toend delay, number of hops, energy consumed and number of alive nodes. The rest of the paper is organized as follows: Section II provides some of the related proposed work. Section III provides a explanation of proposed EEDR routing protocol. Section IV gives the brief study on various path loss models and their mathematical evaluation. In Section V, the results are discussed and Section VI concludes the proposed work.

\section{Related Work}

The work in [9] investigates the environments for different propagation losses in WSNs. The experiments are conducted and the results are examined for different heights of the transceiver. 
This work verifies the inaccuracy of path loss under free space and two ray models in some environments. A new path loss model is presented with realistic measures under the green house environments considering the effects of scattering, reflection and diffraction. The ray tracing simulation results under huge urban environments are presented in [10]. The work considers few kilometers of area around the transmitter with the effects of transmitter height, buildings, streets and statistics like n-values are extracted to obtain the local features. In [11], the spectrum of millimeter wave is proposed for the application in $5 \mathrm{G}$ systems that consumes larger bandwidth. This work derives path loss models by conducting few measurements under outdoor circumstances in the line of sight environments. The shadow effects are obtained from the calculated path loss at the receiver and further the work is carried for wideband channels considering dispersion of time.

The authors of [12] have done a survey on the prediction methods in the path loss. The taxonomy of diversity and resemblance of different approaches are discussed. The signal under transmission gets delayed due to reflections or it will have shift in the frequency due to diffraction and thus the signal fades. The usage of any path loss model in different applications needs a thorough knowledge on the accuracy in real world scenario. Most of the applications use stochastic models (e.g., Hata Model) which consider only minute environmental information, whereas ray models are used with specific calculations under the influence of obstacles at different positions. The work in [13] presents a path loss model based on the probabilistic millimeter wave approach under line of sight conditions. The distance between transmitter and receiver is indicated by weighting function. The proposed model can be used for finding interference, signal coverage estimation in $\mathrm{mm}$-Wave systems. The fifth generation wireless communication system is designed in [14] for micro and macro set-up in urban areas. The close-in free space reference distance model is compared with alpha-beta-gamma model under line of sight environments and non-line of sight environments. The performance parameters such as stability and accuracy are analyzed for a range of millimeter wave and micro wave frequencies using real data.

The authors of [15] propose an adaptable update strategy that wipes out the disadvantages of intermittent beaconing, and a QoS based routing that considers the QoS prerequisites of ongoing applications by ranking the diverse traffics. The advantage of this work is, the performance analysis demonstrates a huge decrease in the utilization of energy and a spread of the traffic among the diverse sensor nodes while ensuring a decent QoS in the traffic. In [16], the authors propose multipath directing strategies to routing protocol for low power and lossy networks to mobile environments. The alternative route is provided for the lower quality links and also provides disjoint routes between the node and the sink. The advantage of this work is, it reduces the network overhead, delay and packet loss.

In [17], the authors propose a solution to mesh network issues by considering both dynamic and static loads of the traffic. It provides an improved performance in various traffic considerations and the results express the competitive performance. The work in [18] proposes an event driven based routing process that reduces the utilization of energy for monitoring the emergency conditions. If the event occurs, then a confined topology is created and the message is transferred through the cluster tree by avoiding more amount of flooding in the network. The advantage of this work is, the lifetime of the network is improved with the reduced energy consumption.

The key concern of the existing state-of-the-art routing methods is they find the routes to the destination when the sender wants to send the data. The proposed protocol considers the requirement of receiver node for receiving data from any of the other node (sender node). In EEDR routing process, multiple routes are discovered with highest channel quality and stored at the sender node's buffer and it uses the alternate route in case of any route failures and hence the route maintenance issues are also reduced.

\section{Energy Efficient Distributed Receiver Based Routing Protocol}

\subsection{Node distribution}

The nodes are deployed in certain area (ex. 1000m X 1000m). The area of deployment and the number of nodes to be chosen depends on the user input. The nodes are able to communicate with only the neighbor nodes which are in its transmission range. Hence all the nodes know their neighbor nodes which are within the transmission range and also nodes having energy level above the threshold level. All the nodes are identified by an ID, and the position of each node is recognized by the triplet formed by the $(X, Y$, Node $I D)$. The $X$ value is the random value between the $X$ min and Xmax. The $Y$ value is the random value between the $Y$ min and Ymax. Here the minimum and maximum values depend on the area of deployment and hence all the nodes are deployed in random.

\subsection{Route discovery and route maintenance}

The Energy Efficient Distributed Receiver (EEDR) based routing protocol [8] for WSNs starts the route discovery from the receiver side to the sender side. The algorithm shows the implementation of EEDR routing protocol.

\section{ALGORITHM:}

EEDR Routing Protocol implementation

1. Nodes are distributed in random

2. The receiver node sends the Call to Relay Node $(C R N)$ to all its neighboring nodes

3. The neighbor/intermediate nodes receive the CRN packet.

4. The node checks whether the CRN is received before by verifying Broadcast ID

5. Drop packet if it is received before, else check whether CQI is greater than threshold value

6. If CQI is less than threshold then drop packet, else update the CQI and forward the CRN to neighbor nodes till the sender is reached.

7. When the CRN reaches the sender, Update CQI and send Data to receiver on best route

The nodes are deployed in random and among the nodes, one is chosen as Receiver (R) and one node as Sender (S) as in Fig.1. The route discovery starts with the broadcast of Call to Relay Node (CRN) from the receiver to its neighbors as in Fig.2. The CRN packet consists of information such as <receiver node ID, receiver sequence number, CQI, sender node ID, sender sequence number, hop count $>$. The intermediate nodes that receive the CRN packets will check for the broadcast ID to avoid retransmissions and looping. If the broadcast ID is same, then the CRN packet will be dropped otherwise the Channel Quality Indicator (CQI) is calculated. For considering any stable routing, the channel quality must be high. The intermediate node being the sender node is a possibility and if the node is the sender and the CQI is better than before, then data from the sender follows the new best route to the receiver, otherwise the CQI is just updated and the $\mathrm{CRN}$ is 
forwarded further until it reaches the sender node as in Fig 3(a), 3(b) and 3(c).

The CQI values are calculated from the SNIR values of the received CRN packet. CQI is calculated by (1) and SNIR is calculated by (2).

$C Q I=\left\{\begin{array}{l|c}\left\lfloor\frac{S N I R}{0}+16.62\right. \\ 30\end{array}\right] \begin{gathered}S N I R \leq-16 \\ -16<S N I R<14 \\ 14 \leq S N I R\end{gathered}$

$S N I R=\frac{\frac{P_{T}}{L_{P}}}{N_{0} W} \times P G$

Where,

$P_{T}=$ Transmitted Power,

$L_{P}=$ Path Loss,

$W=$ Signal bandwidth

$N_{0}=1.38 * 10^{-23} * 290$.

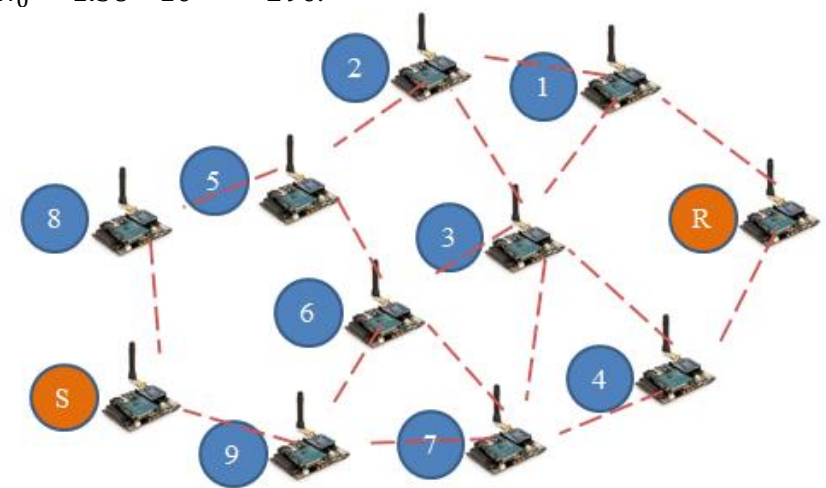

Fig.1: Distribution of sensor nodes.

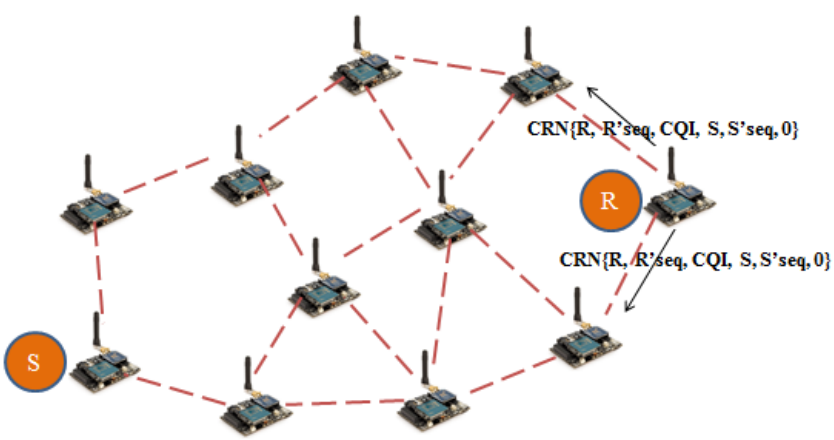

Fig.2: Receiver node initiates route discovery.

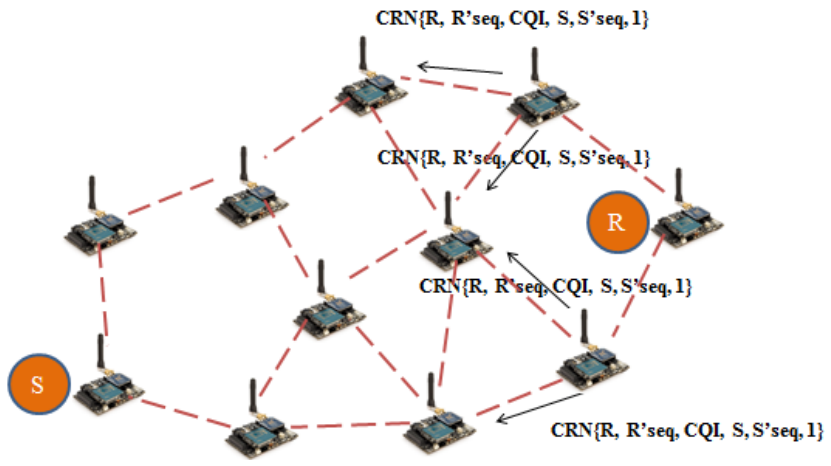

Fig. 3(a): Route establishment.

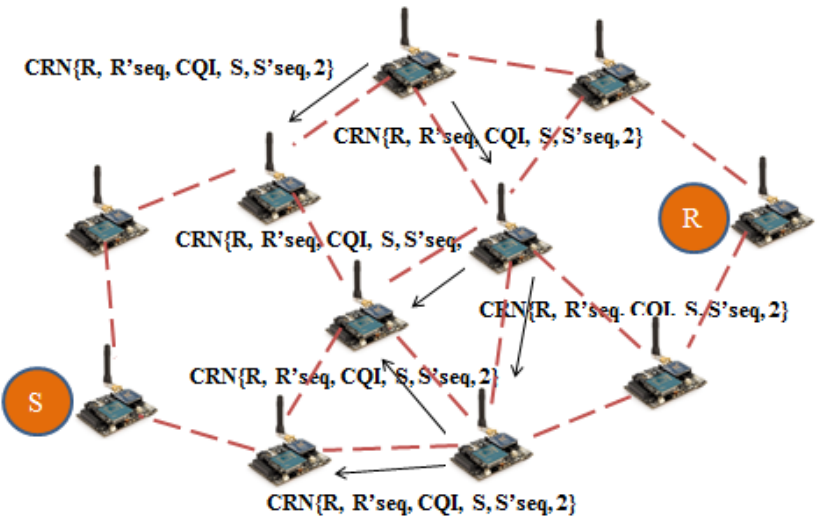

Fig. 3(b): Route establishment

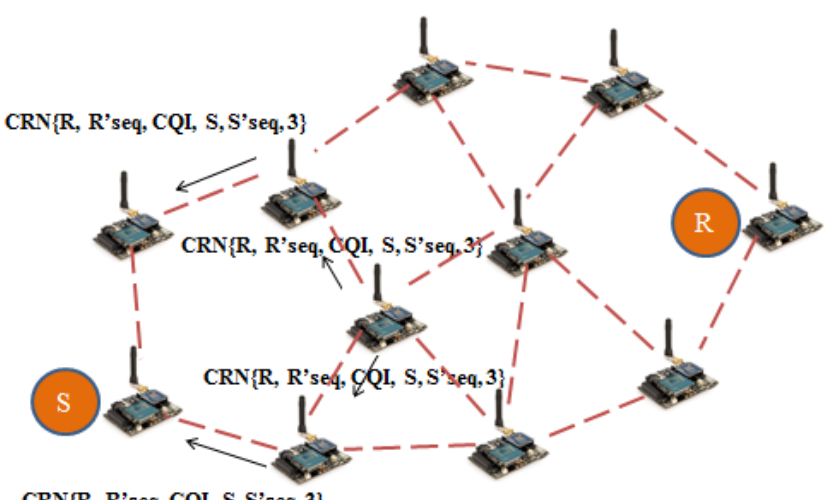

CRN\{R, R'seq, CQI, S, S'seq, 3\}

Fig. 3(c): Route establishment

Fig. 3: Route discovery between receiver and sender.

The sender will know the received path quality by the CQI and if the channel is good then it starts sending the data to the receiver through that path. The sender may still receive the CRN packets from different paths when it has already initiated the data transmission as in Fig. 4(a). The sender stores the multiple paths in its buffer if the channel quality is good. The data is transmitted to the receiver as in Fig. 4(b). The receiver will send the Acknowledgement (ACK) to the sender as a confirmation to received data as in Fig. 4 (c).

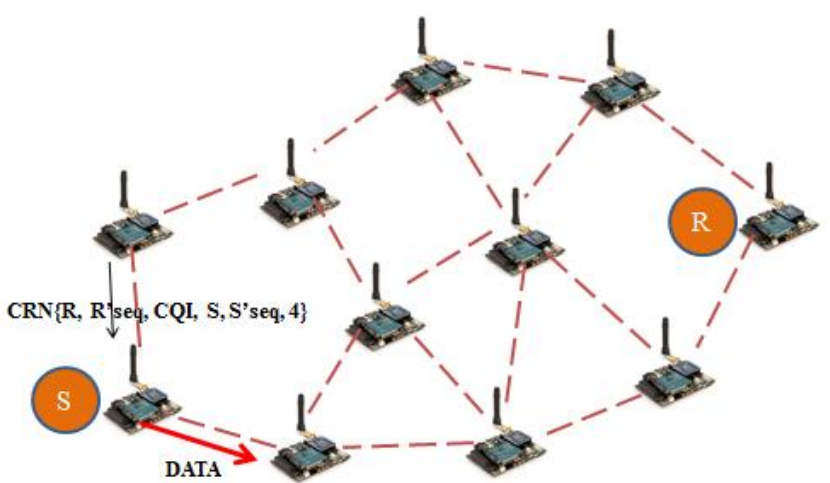

Fig. 4(a): Data Transfer 


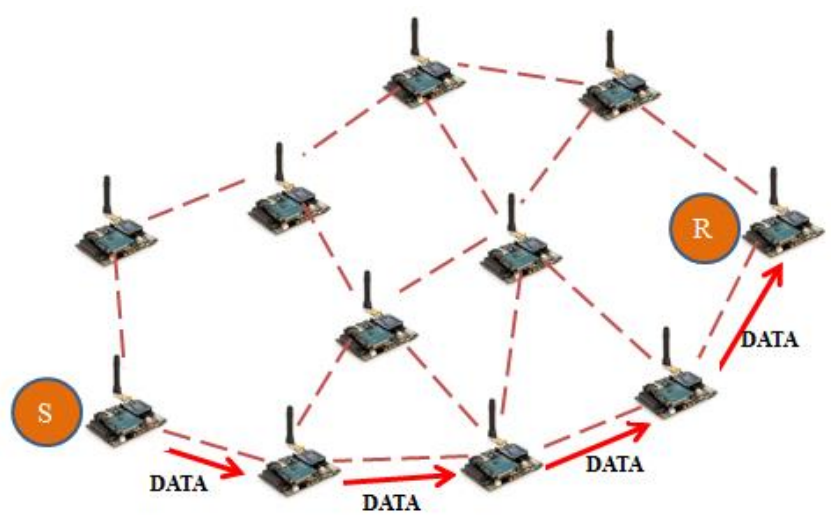

Fig. 4(b): Data transmission

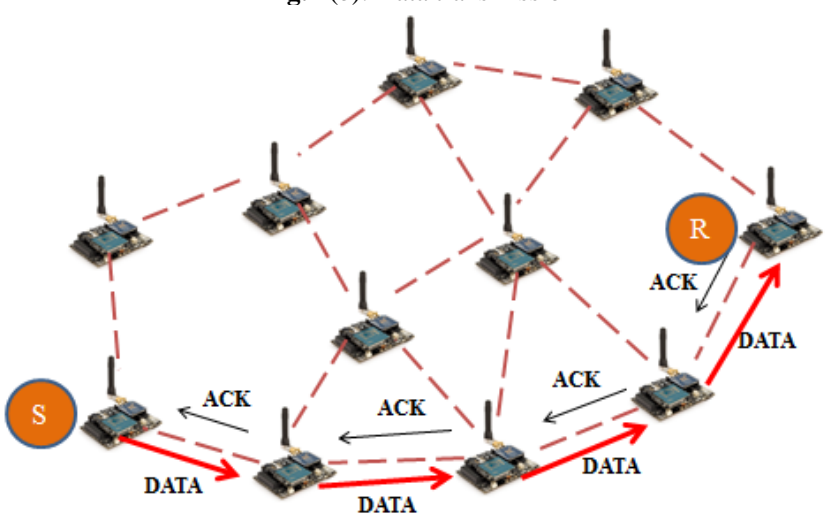

Fig. 4(c): Acknowledgement for data

Fig. 4: Data transmission from sender to receiver.

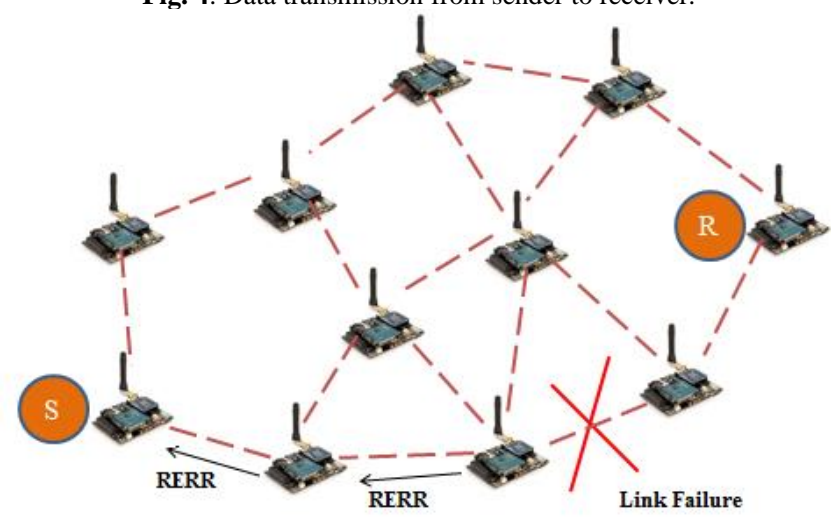

Fig. 5: Link failure and route error.

There are chances of link failure in the network because of the mobility of the nodes and node failure because of decrease in the energy levels of the nodes. In such case, the sender will receive a Route Error (RERR) message as in Fig. 5. The sender node chooses the next best route from its buffer to send the data to the receiver as in Fig. 6(a) and receives the ACK for the same as in Fig 6 (b).

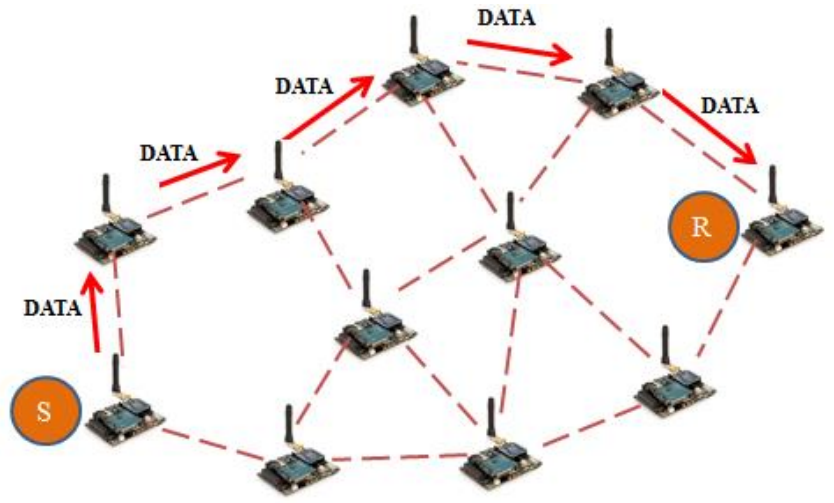

Fig. 6(a): Data transmission in alternate route

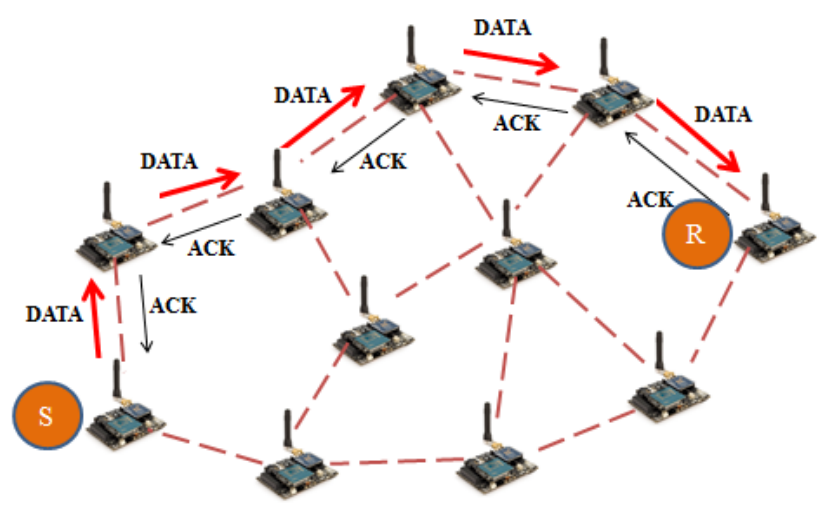

Fig. 6(b): Acknowledgement form alternate route

Fig. 6: Data transmission in new route.

If there are no more requests from other nodes for route discovery or if the node is not involved in any data transmission then the node will go for sleep mode for saving battery power.

\section{Path Loss Models}

The path loss is also referred to as attenuation. It is the reduction of the power when the data is transmitted from the sender to the receiver. Path loss is of major consideration for the analysis of signal propagation in wireless communication system. As the path loss is influenced by various environmental constraints, different path loss models are considered here indicating different environmental conditions for the propagation of the signals where the major path loss takes place. The Okumura-Hata model is considered as it predicts the path loss under the external environments in the cellular transmissions with high frequency ranges. The Okumura path loss model is considered for large and medium cities with highly built up areas. The Walfisch-Ikegami model is one of the popular path loss models which consider the reflection and diffraction. The other important path loss models considered are the clutter factor model and micro cell path loss model. The clutter factor model consists of the plane earth and extra factor based upon a large number of measurements. The micro cell path loss model considers the large number of users in small area covering few hundred meters by reuse of frequency at short distances. The evaluation of the path loss models is as below:

\subsection{Okumura-Hata model}

Okumura-Hata [19] model is one of the most popular path loss models which has the frequency ranging from $150 \mathrm{MHz}$ to $1500 \mathrm{MHz}$. The height of the transmitter varies from 30 to $200 \mathrm{~m}$; the height of the receiver varies from 1 to $10 \mathrm{~m}$. The distance between the transmitter and receiver is between 1 and $10 \mathrm{Km}$. 
There are different prediction areas under Okumura-Hata model; (i) Open area, where there are no tall buildings or tall trees in the path. (ii) Suburban area, where it is village environment and it may have some obstacles in covered area but not too congested. (iii) Urban area, where it has tall buildings and trees and more obstacles under the covered area. The path loss for Hata model is calculated as below

$$
\begin{aligned}
& \text { For urban areas: } L_{d B}=A+B \log _{10}(R)-E \\
& \text { Suburban areas: } L_{d B}=A+B \log _{10}(R)-C \\
& \text { Open areas: } L_{d B}=A+B \log _{10}(R)-D \\
& A=69.55+26.16 \log _{10}\left(f_{c}\right)-13.82 \log _{10}\left(h_{b}\right) \\
& B=44.9-6.55 \log _{10}\left(h_{b}\right) \\
& C=2\left(\log _{10}\left(f_{c} / 28\right)\right)^{2}+5.4 \\
& D=4.78\left(\log _{10} f_{c}\right)^{2}+18.33 \log _{10} f_{c}+40.94 \\
& E=3.2\left(\log _{10}\left(11.7554 h_{m}\right)\right)^{2}-4.97
\end{aligned}
$$

\subsection{Walfisch-Ikegami model}

The Walfisch-Ikegami [20] model considers the city structure characteristics such as building heights, road widths, road orientations, separation between the buildings. This model is little complex and it produces accuracy in the estimation of the propagation. The range of operation of frequency is from $800 \mathrm{MHz}$ to $2000 \mathrm{MHz}$. The transmitter height varies from $4 \mathrm{~m}$ to $50 \mathrm{~m}$; the height of the receiver varies from $1 \mathrm{~m}$ to $3 \mathrm{~m}$. The distance between the transmitter and the receiver is between 0.02 and $5 \mathrm{Km}$.

The path loss for Walfisch-Ikegami model is given by

$L_{d B}=42.6+26 \log _{10}(d)+20 \log _{10} f_{c}$

Where,

$d=$ Distance

$f_{c}=$ Carrier frequency in $\mathrm{MHz}$

\subsection{Clutter Factor model}

Clutter Factor [21] model considers the reflections from the ground. It has different approaches such as electromagnetic solving which is very complicated and used under ray tracing and requires more computer operation. It considers the reflection and refraction of the signals, it also considers the diffraction if the frequency is less than 10GHz. The path loss for Clutter Factor model is given by

$L_{d B}=40 \log _{10}(r)-20 \log _{10}\left(h_{m}\right)-20 \log _{10}\left(h_{b}\right)$

Where,

$r=$ distance between the nodes

$h_{b}=$ node $b$ antenna height

$h_{m}=$ node $m$ antenna height

\subsection{Micro cell path loss model}

Micro cell path loss [22] model is a dual slope path loss model, where the propagation characterization considers two different path loss exponents and the breakpoint distances. The breakpoints are maintained at the distance of few meters. The path loss for Micro cell path loss model is given by

$$
\begin{gathered}
L_{d B}=10 n_{1} \log _{10}(r)+L_{1}, \text { for } r<r_{b} \\
\begin{array}{c}
L_{d B}=10 n_{2} \log _{10}\left(r / r_{b}\right)+10 n_{1} \log _{10}\left(r_{b}\right)+L_{1}, \text { for } r \\
>r_{b}
\end{array}
\end{gathered}
$$

Where,

$L_{1}=$ reference path loss at $r=1 \mathrm{~m}$

$r_{b}=$ breakpoint distance

$n_{l}=$ path loss exponent for $r \leq r_{b}$

$n_{2}=$ path loss exponent for $r>r_{b}$

\section{Results}

\subsection{Simulation Test bed}

The proposed work is evaluated using the MATLAB software. Initially $n$ number of nodes is randomly deployed in certain user defined area. The nodes are set to have certain transmission range for communication and all the nodes are assumed to have same energy levels. Among the deployed nodes, one node is chosen as sender and one node is chosen as receiver. The route establishment is initiated from the receiver side to find the path to the sender. The route establishment considers the energy consumption for packet transmission. The route establishment is done between the same sender and the receiver under different path loss models and the performance comparison is made considering end-to-end delay, number of hops, energy consumption and number of alive nodes. In the simulation of proposed EEDR routing protocol, some of the assumptions are made with reference to routing. The assumed parameters are based on the user inputs and the parameters are as below:

1. Area of deployment

2. Number of nodes in the network (randomly deployed)

3. Sender node and receiver node

4. Initial energy of nodes

5. Number of iterations of execution

6. $\quad$ Energy required for transmission

7. Attenuation factor

8. Time to leave value

\subsection{Performance analysis}

The EEDR protocol is executed under various path loss models to check its performance in different environments. The simulation parameters for the performance evaluation are shown in TABLE I. The simulation tool used is MATLAB. The number of nodes chosen is 100 and the topology area is considered to be $1000 \mathrm{~m} \mathrm{X}$ $1000 \mathrm{~m}$. The nodes are deployed in random [22]. The protocol is executed for 5 iterations with each node having transmission range of $200 \mathrm{~m}$. The energy required for transmission is taken as $15 \mathrm{~mJ}$ and the energy required for amplification is taken as $5 \mathrm{~mJ}$. The attenuation factor is 0.5 and the initial battery level of nodes is chosen as infinity and the time to leave (TTL) is taken as 4 . 


\begin{tabular}{|c|c|}
\hline Parameter Name & Value \\
\hline Number of Nodes & 100 \\
\hline Initial Energy of nodes & $3000 \mathrm{~mW}$ \\
\hline Topology area & $1000 \mathrm{~m} \mathrm{x} 1000 \mathrm{~m}$ \\
\hline Number of Iterations & 5 \\
\hline Transmission Range & $1-200 \mathrm{~m}$ \\
\hline Energy required for transmission & $15 \mathrm{~mJ}$ \\
\hline Attenuation Factor & 0.5 \\
\hline Sender Node & 60 \\
\hline Receiver Node & 77 \\
\hline Time to leave & 4 \\
\hline
\end{tabular}

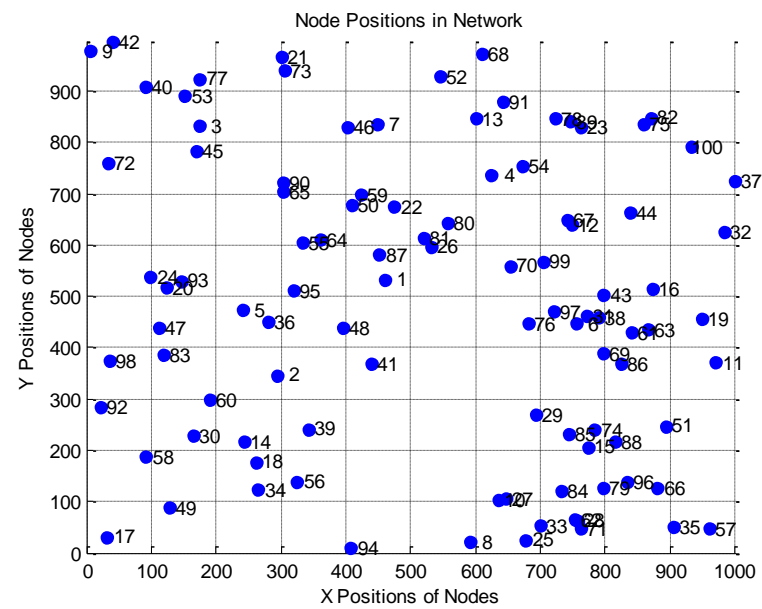

Fig. 7: Distribution of nodes in the network.

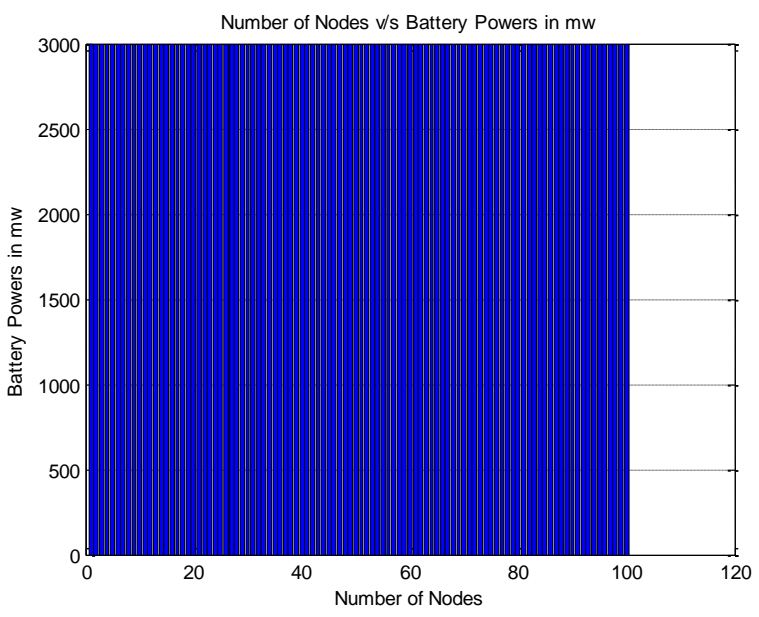

Fig. 8: Initial Energy of nodes

The nodes are distributed in random as in Fig. 7. The initial energy of all nodes is assumed to be $3000 \mathrm{~mW}$ as in Fig. 8 .

\subsubsection{End-to-end delay}

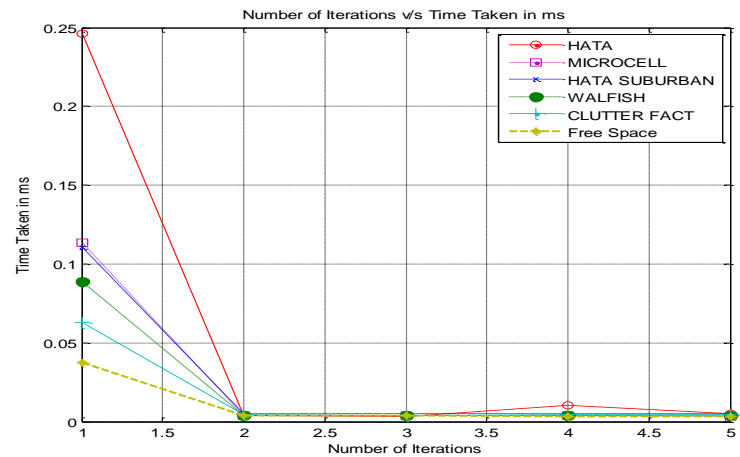

Fig. 9: End-to-end delay
The end to end delay is the time taken by data packet to reach the destination. This time covers the time required for computation of the route and queue time. The EEDR protocol has very less endto-end delay than existing reactive and proactive routing protocols [8]. Fig. 9 shows the time taken by various path loss models to deliver the data packet from source to destination. During the first iteration, all the path loss models takes little time for route discovery and hence consumes more time than all other iterations in delivering the packets. The delay time reduces from the second iteration onwards.

\subsubsection{Number of hops}

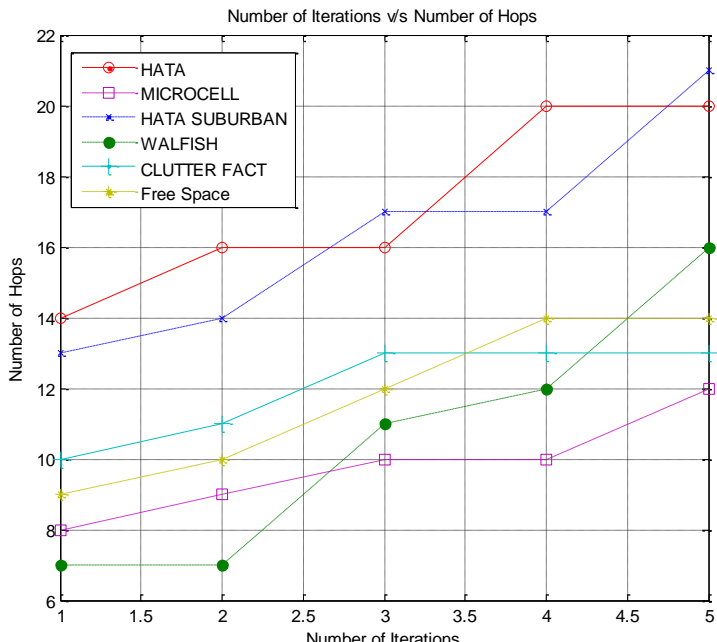

Fig. 10: Number of hops

Number of hops indicates the total number hops taken by the data packet to travel from the sender to the receiver. After the route establishment from the receiver side, the sender will select the best route from all the routes discovered based on the quality of the channel. The EEDR protocol takes less number of hops than existing reactive and proactive routing protocols [8]. Fig. 10 shows the number of hops taken by different path loss models, the number of hops should be kept minimal for reduction in the consumption of the energy.

\subsubsection{Energy consumption}

The energy consumption in the network is due to the establishment of the route and maintenance of the route. The energy consumption should be minimized in order to improve the network life time. The EEDR protocol consumes less amount of energy than existing reactive and proactive routing protocols [8]. Fig. 11 shows the performance of EEDR protocol for the various path loss models. The consumption of energy slightly increases as the number of iterations is increased. 


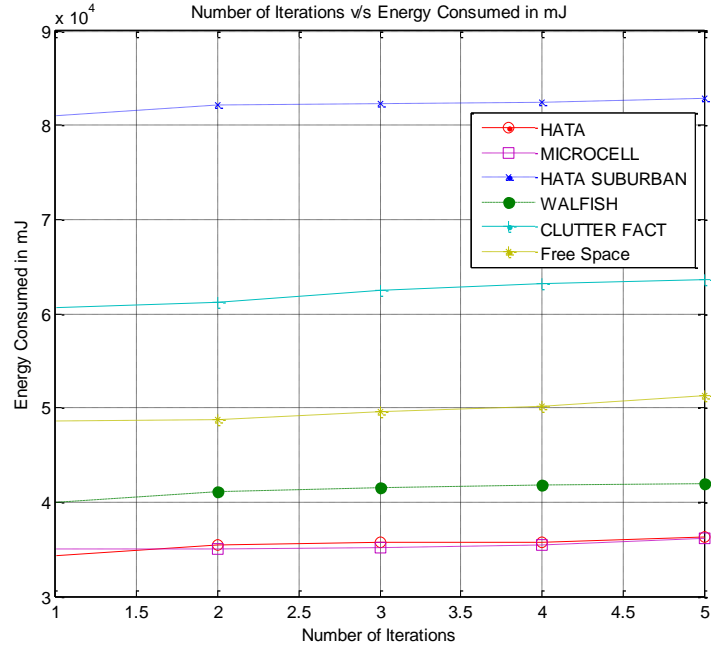

Fig. 11: Energy consumed

\subsubsection{Number of alive nodes}

The nodes in the network have limited amount of battery power and hence they have to maintain their energy for longer time to be active. The node is said to be dead if its energy level of the node goes below the threshold level. The EEDR protocol has more number of alive nodes than the existing reactive and proactive routing protocols [8]. Fig. 12 shows the number of alive nodes in the network for each iteration. The energy consumption in the network is because of the route establishment, route maintenance and the data transmission. The nodes which involve the data transmission consume more energy than other nodes. As the number of iterations increases, the nodes which involve in the data transmission will have their energy less than the minimum threshold energy level. Hence the number of alive nodes decreases with increase in number of iterations.

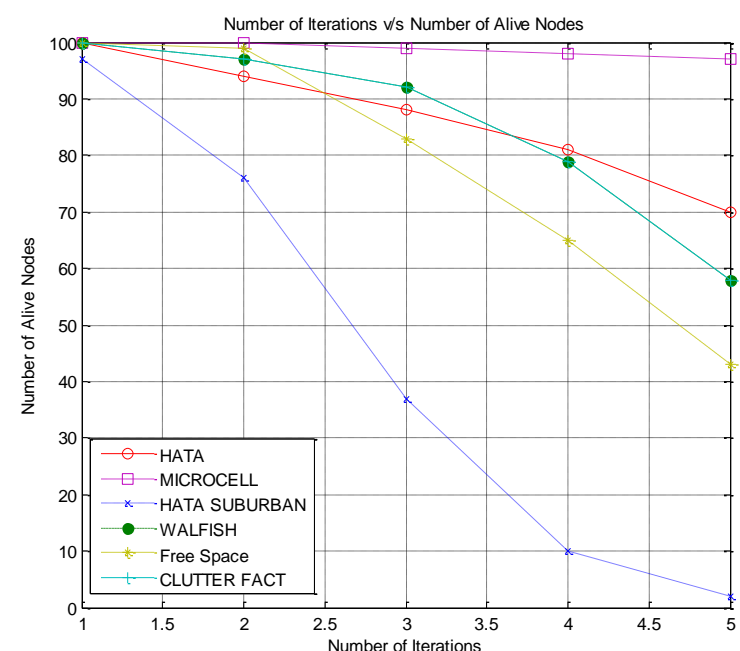

Fig. 12: Number of alive nodes

\section{Conclusion}

The path loss is an important parameter to be considered for evaluation of any protocol in wireless sensor networks. It is difficult to practically measure and evaluate any wireless protocol in various environmental conditions. The path loss models help in evaluating the wireless communication protocol theoretically and analyze the characteristics in different environments. In this paper a brief study on different path loss models is provided and path loss models are mathematically analyzed. The proposed work also evaluates the EEDR routing protocol for end-to-end delay, number of hops, energy consumption and the number of alive nodes under various path loss models. From the simulation results, it is observed that, the path loss varies for different environments and hence we see the variation in routing performance in wireless networks.

\section{References}

[1] Yick, Jennifer, Biswanath Mukherjee, and Dipak Ghosal, "Wireless sensor network survey", ELSEVIER Computer networks, Vol. 52, Issue 12, pp. 2292-2330, August 2008.

[2] Rault T, Bouabdallah A, Challal Y, “ Energy efficiency in wireless sensor networks: A top-down survey", ELSEVIER Computer Networks, Vol. 67, pp. 104-22, July 2014.

[3] Mahmood, Muhammad Adeel, Winston KG Seah, and Ian Welch, "Reliability in wireless sensor networks: A survey and challenges ahead", ELSEVIER Computer Networks, Vol. 79, pp. 166-187, March 2015.

[4] Zhang, Xinchen, and Jeffrey G. Andrews. "Downlink cellular network analysis with multi-slope path loss models." IEEE Transactions on Communications, Vol. 63, Issue 5, pp.1881-1894, 2015.

[5] Aremu, O. A., Taiwo, O. A., Makinde, O. S., \& Adeniji, J. A., "Experimental Study of Variation of Path Loss with Respect to Heights at GSM Frequency Band", International Journal of Scientific Research in Science, Engineering and Technology, Vol. 2, Issue 3, 2016.

[6] Zhang, Bei, Zhangdui Zhong, Xin Zhou, Ke Guan, and Ruisi He., "Path loss characteristics of indoor radio channels at $15 \mathrm{GHz}$ ", 2016 10th European Conference on Antennas and Propagation (EuCAP), pp. 1-5. IEEE, DOI: 10.1109/EuCAP.2016.7481177, 2016.

[7] Herring K T, Holloway J W, Staelin D H, Bliss D W, "Path-loss characteristics of urban wireless channels", IEEE Transactions on Antennas and Propagation, VOL. 58, Issue 1, JANUARY 2010.

[8] Pramod M S, Shivashankar, "Performance analysis of EEDR routing protocol for WSNs", IET Wireless Sensor Systems, Vol. 7, Issue 1, pp 21-26, ISSN 2043-6386, doi: 10.1049/ietwss.2016.0029, September 2016.

[9] Raheemah, A., Sabri, N., Salim, M. S., Ehkan, P and Ahmad, R. B., "New empirical path loss model for wireless sensor networks in mango greenhouses", ELSEVIER Computers and Electronics in Agriculture, Vol.127, pp. 553-560, September 2016.

[10] Green Daisy, Zhengqing Yun and Magdy F. Iskander, "Propagation characteristics in urban environments", IEEE/ACES International Conference on Wireless Information Technology and Systems (ICWITS) and Applied Computational Electromagnetics (ACES), DOI: 10.1109/ROPACES.2016.7465342, March 2016.

[11] Al-Samman, A. M., Rahman T. A., Azmi, M. H., Zulkefly N. R and Mataria, A. M, "Path loss model for outdoor environment at 17 $\mathrm{GHz}$ mm-wave band", IEEE 12th International Colloquium on Signal Processing \& its Applications (CSPA2016), pp. 179-182, DOI: 10.1109/CSPA.2016.7515827, March 2016.

[12] Phillips Caleb, Douglas Sicker, and Dirk Grunwald, "A survey of wireless path loss prediction and coverage mapping methods", IEEE Communications Surveys \& Tutorials, VOL. 15, Issue. 1, pp. 255-270, 2013.

[13] Samimi. Mathew K., Theodore S. Rappaport and George R. MacCartney, "Probabilistic omnidirectional path loss models for millimeter-wave outdoor communications", IEEE Wireless Communications Letters, Vol. 4, Issue 4, pp. 357-360, August 2015.

[14] Sun, Shu, Theodore S. Rappaport, Sundeep Rangan, Timothy A. Thomas, Amitava Ghosh, Istvan Z. Kovacs, Ignacio Rodriguez, Ozge Koymen, Andrzej Partyka, and Jan Jarvelainen, "Propagation path loss models for $5 \mathrm{G}$ urban micro-and macro-cellular scenarios", IEEE 83rd Vehicular Technology Conference, DOI: 10.1109/VTCSpring.2016.7504435, May 2016.

[15] Ziad Serhan and Wafaa Bou Diab, "Energy efficient QoS routing and adaptive status update in WMSNs", Inderscience PublishersInternational Journal of Space-Based and Situated Computing, Vol. 6, Issue 3, pp. 129-146, 2016.

[16] Fatma Somaa, InÁ"s El Korbi and Leila Azouz Saidane, "Proactive vs. reactive multipath routing for mobility enabled RPL", Inderscience Publishers-International Journal of Space-Based and Situated Computing, Vol.7, Issue 2, pp. 94-107, 2017. 
[17] Waheed, H.J., Abduljalil, M., Alkuraishy, H. "Estimation of apolipoprotein A, apo B, apo E and somebiochemical markers in type 2 diabetic patients in Iraq",(2018) International Journal of Pharmaceutical Research, 10 (3), pp. 493-498.

[18] Jing Zhang, Ting Yang and Chengli Zhao, "Energy-efficient and self-adaptive routing algorithm based on event-driven in wireless sensor network", Inderscience Publishers-International journal of grid and utility computing, Vol.7, Issue 1, pp.41-49, 2016.

[19] Keawbunsong P, Promwong S, "Comparison study on path loss model for DVB-T2 propagation in urban area", IEEE Conference on Antenna Measurements \& Applications (CAMA), pp.1-4, DOI: 10.1109/CAMA.2015.7428133, November 2015.

[20] Katev P D, "Propagation models for WiMAX at $3.5 \mathrm{GHz}$, IEEE ELEKTRO, pp. 61-65, DOI: 10.1109/ELEKTRO.2012.6225572, ISBN: 978-1-4673-1179-3, May 2012.

[21] A.A.Zavala, Simon.R.S, "Antennas and propagation for wireless communication systems", Second Edition, John Wiley \& Sons, ISBN 978-0-470-84879-1, Sep 2008.

[22] Pramod M S, Shivashankar, Mohammed Khurram, "Random node deployment and route establishment in receiver based routing protocol for WSNs", 2016 IEEE International Conference on Recent Trends in Electronics, Information \& Communication
Technology
(RTEICT)
pp. $\quad 74-78$

DOI: 10.1109/RTEICT.2016.7807786, 2016 